\title{
Modelos de modernización para el desarrollismo: el influjo de las propuestas estadounidenses en el Servicio de Extensión Agraria (1955-1975) ${ }^{1}$
}

\author{
Alba DíAz-GEADA \\ Universidade de Santiago de Compostela \\ alba.diaz@usc.es \\ Daniel LANERO TÁBOAS \\ Universidade de Santiago de Compostela \\ daniel.lanero@usc.es
}

Recepción: 10 de febrero de 2015 / Revisión: 5 de abril de 2015

Aceptación: 18 de abril de 2015 / Publicación: Diciembre de 2015

\begin{abstract}
RESUMEN
Con este trabajo nos proponemos estudiar las políticas agrarias del franquismo y particularmente la creación y actuación del Servicio de Extensión Agraria (1955) en el contexto de la difusión de la Teoría de la Modernización. Comenzaremos con una contextualización de las relaciones culturales entre España y los EE.UU. y su potencial proyección sobre el ámbito rural. A continuación, prestaremos atención a la impronta del modelo extensionista norteamericano en la organización y metodología del Servicio de Extensión Agraria. Sin perder de vista la importancia de la escala estatal para la implementación y adaptación de las distintas políticas agrarias, atenderemos al contexto político e ideológico internacional como elemento que permite ampliar la comprensión y estudio de dichas políticas. En ese sentido, propondremos finalmente una primera aproximación comparativa al estudio del SEA en relación con diferentes experiencias de extensionismo agrario en Latinoamérica.
\end{abstract}

Palabras clave: Teoría de la Modernización, Guerra Fría, extensionismo agrario estadounidense, Servicio de Extensión Agraria, franquismo, América Latina, España, Estados Unidos, siglo XX.

\section{Modernization Models for Development Policy: American Proposals and their influence on the Servicio de Extensión Agraria (1955-1975)}

\begin{abstract}
This article proposes the study of Francoism's agrarian policies and, in particular, the creation and operations of the Servicio de Extensión Agraria (Agrarian Extension Service, 1955), within the context of the diffusion of the Modernization Theory. The article begins by contextualizing the cultural relations between Spain and the United States and their potential projection into the rural sphere. It goes on to focus on the mark left by the United States' extensionist model on the organization and methodology of the Servicio de Extensión Agraria. Without disregarding the importance of the state level in the implementa-
\end{abstract}

1 Este trabajo se ha beneficiado de la financiación del Proyecto de Investigación del MINECO: HAR2013 - 47934 - P: "La gran transformación tecnológica y social de las agriculturas ibéricas. Viejos, nuevos y novísimos paradigmas, 1950-2000”. IP: Lourenzo Fernández Prieto. 
tion and adaptation of the different agrarian policies, the international political and ideological context is also addressed as an element that allows for a broader comprehension and study of said policies.

Keywords: Modernization Theory, Cold War, Agrarian Extension in the United States, Servicio de Extensión Agraria, Francoism, Latin America, Spain, United States, $20^{\text {th }}$ Century.

SUMARIO: 1. Introducción. 2. Una aproximación a las relaciones políticas y culturales entre España y EE.UU. a partir de 1945. 3. La influencia del modelo norteamericano en el extensionismo agrario en España (1955-1975). 4. El Servicio de Extensión Agraria (SEA) español en el marco del extensionismo internacional de posguerra. 5. Conclusiones. 6 . Referencias bibliográficas.

\section{INTRODUCCIÓN}

Durante las décadas de los 50 y los 60, en el contexto geopolítico de la Guerra Fría y en el marco de la tensión ideológica de un mundo concebido bipolarmente, se fue configurando la llamada Teoría de la Modernización. Se trata de una teoría del desarrollo inscrita en un posicionamiento conceptual dicotómico, dividiendo a las sociedades en "modernas" y "tradicionales". Partiendo de esas dos categorías, las sociedades tradicionales llegarían al estadio de las modernas, de acuerdo a los parámetros de inteligibilidad establecidos desde fines del siglo XIX. En ese contexto, la Teoría de la Modernización, intentó sentar parámetros para regular ese proceso, entendido como inevitable. Esta teoría legitimaba un papel activo de los Estados en el impulso a este proceso, así como un papel director de los EE.UU. como potencia capitalista hegemónica en la promoción e implementación de medidas que favoreciesen un determinado modelo de desarrollo, de acuerdo a parámetros occidentales y de acuerdo a las necesidades de contención política de otros modelos alternativos. Tanto el Plan Marshall en Europa como la Alianza para el Progreso en Latinoamérica fueron iniciativas influidas por esta teoría ${ }^{2}$.

En el contexto de la segunda posguerra mundial, comenzó a gestarse asimismo la denominada, desde finales de los sesenta, Revolución Verde. Sobre la base de la necesidad de acabar con el problema del hambre en el mundo (y su corolario de conflicto político) como argumento legitimador, se promovieron en diferentes países bajo la órbita de los EE.UU. medidas de tecnificación y especialización agraria para un aumento de la productividad, a costa de la pérdida de autonomía de las diferentes agriculturas históricas ${ }^{3}$.

A comienzos de los cincuenta la dictadura franquista suscribió un acuerdo de colaboración militar y económica con los EE.UU. (Pactos de Madrid, 1953), que tuvo consecuencias en la política agraria del régimen. Nuestro propósito con este trabajo es estudiar la creación y actuación del Servicio de Extensión Agraria (1955) en el contexto de la difusión de la Teoría de la Modernización en el ámbito agrario.

2 Sobre la Teoría de la Modernización: Rostow, 1961; Huntington, 1968, 1976; Apter, 1972a, 1972b; BENDIX, 1966-1967; para el ámbito rural: RoGERs, 1969.

3 Shiva, 1991; Picado, 2011, pp. 25-50. También Picado, 2012b, pp. 107-134. 
La extensión agraria puede ser concebida de diferentes formas en función de la teoría de desarrollo a la que vaya asociada. En el contexto en que nos situamos, la teoría de desarrollo dominante estaba permeada por determinadas ideas de modernidad y progreso, que llegaron a ser consideradas como equivalentes. La extensión funcionaría como una herramienta de transmisión de conocimiento que, en conexión con otras políticas agrarias, mediaba entre el entramado institucional e investigador y la estructura social y cultural que se pretendía modificar ${ }^{4}$.

Comenzaremos el trabajo con una contextualización de las relaciones culturales entre España y los EE.UU., particularmente en el ámbito agrario, en el marco de la hegemonía estadounidense que caracterizó el mundo de posguerra. A continuación, prestaremos atención a la impronta del modelo extensionista norteamericano en la organización y metodología del Servicio de Extensión Agraria. Sin perder de vista la importancia del contexto histórico concreto, tanto del conjunto estatal como de los distintos ámbitos locales, para la comprensión de la implementación y adaptación de las distintas políticas agrarias, en esta ocasión trataremos de poner énfasis en la importancia del contexto político e ideológico internacional como elemento que permite ampliar la comprensión y estudio de dichas políticas.

Para finalizar, propondremos un enfoque comparado con el extensionismo agrario de algunos países latinoamericanos. Si bien cada proceso nacional tiene una especificidad que nace de circunstancias propias del modo y momento en que se dio el influjo de la Teoría de la Modernización, así como de los actores involucrados, es preciso subrayar el carácter expansivo de una lógica donde los niveles de intervención estatal y penetración de capital extranjero apuntaban a la modificación de la infraestructura económica ${ }^{5}$.

\section{UNAAPROXIMACIÓN A LAS RELACIONES POLÍTICAS Y CULTURALES ENTRE ESPAÑA Y EE.UU. A PARTIR DE 1945}

La historiografía sobre las relaciones políticas entre el régimen de Franco y las diferentes administraciones norteamericanas ha experimentado un importante desarrollo en la última década ${ }^{6}$. Durante la Segunda Guerra mundial y en los primeros años de la posguerra, las relaciones entre ambos Estados se vieron dificultadas por la proximidad del franquismo a las potencias fascistas ${ }^{7}$. Posteriormente, la dinámica de Guerra Fría establecida entre los EE.UU. y la URSS incrementó las opciones de supervivencia política del régimen franquista en el medio y largo plazo, al tiempo que posibilitó la progresiva consolidación de las relaciones bilaterales entre España y los EE.UU. El viraje en la política exterior de los EE.UU. hacia España y el inicio del proceso

\footnotetext{
4 Sánchez De Puerta, 1996, pp. 35-68.

5 Una primera versión de este trabajo en Díaz-GEAdA - LANERO, $2014 \mathrm{~b}$.

6 Delgado - Elizalde, 2005; Viñas, 2003; Niño, 2009, pp. 13-221; Delgado - León, 2011, pp. 5-70; Niño - Montero, 2012; Termis, 2005; Delgado, 2003, pp. 35- 59.

7 De hecho, el antiamericanismo fue la actitud dominante entre buena parte de las elites políticas de la dictadura franquista durante la Segunda Guerra mundial y en los años inmediatamente posteriores. Véase RODRÍGUEZ JiMÉNEZ, 2010.
} 
de aceptación y reconocimiento internacional del régimen, motivaron la búsqueda de soluciones legislativas que permitieran la prestación de ayuda económica a la dictadura de Franco ${ }^{8}$.

En este contexto, los estudios realizados hasta el momento sobre las líneas de acción política y cultural que los EE.UU. desplegaron en España, parecen indicar que la atención prestada a la sociedad rural, por entonces claramente predominante en términos demográficos, fue muy secundaria.

Una de las principales herramientas empleadas durante la Guerra Fría para la difusión de una imagen positiva de la sociedad y el modo de vida americano entre la opinión pública de Europa occidental fue el Foreign Leader Program (FLP) ${ }^{9}$. En España también se puso en marcha, en 1952, este Programa, directamente vinculado en la Península Ibérica a la consecución de los objetivos militares norteamericanos. Los "líderes" españoles invitados a viajar a los EE.UU. se seleccionaron entre las elites políticas y sociales de las principales ciudades del país. Sin embargo, las elites rurales (terratenientes y medianos propietarios agrarios) carecían de valor instrumental para la consecución de los objetivos inmediatos de los EE.UU. en España ${ }^{10}$. Tampoco parece que los burócratas con responsabilidades sobre la política agraria del régimen hayan sido un objetivo prioritario sobre el que ejercer influencia, al menos a través del FLP, si bien en este caso nuestra mirada tenga probablemente que dirigirse a los programas de asistencia técnica.

La situación parece haber cambiado a partir de la década de 1960, cuando garantizar un final no traumático (en términos de cambio político) de la dictadura franquista se convirtió en una prioridad estratégica para la política exterior de los EE.UU. De ahí la necesidad de una modernización acelerada de las estructuras económicas y sociales españolas, y por supuesto del sector agrario ${ }^{11}$.

8 Según ViÑAS, aunque España entraba finalmente (en 1953) en el círculo de países receptores de ayuda económica norteamericana, lo hacía demasiado tarde, cuando la ayuda masiva de los EE.UU. a Europa se estaba agotando y la Organización Europea de Cooperación Económica (OECE) abandonaba los grandes programas de reconstrucción global para centrarse en la búsqueda de vías endógenas que asegurasen la continuidad del crecimiento económico (acuerdos comerciales multilaterales). VIÑ̃S, 2003, p. 284. Para una aproximación más en detalle a este tema, véase GuiRAo, 1998, pp. 131-188.

9 Sobre el Foreign Leader Program, véase: Sсотт-Sмітн, 2012, pp. 123-154. Durante los años cincuenta los beneficiarios del Programa de Líderes fueron casi en exclusivo ciudadanos de países de Europa occidental. El cambio en las prioridades de la política exterior norteamericana en el curso de la Guerra Fría extendieron en la década de 1960 el FLP a América Latina, África, Asia... Una perspectiva global sobre la diplomacia cultural estadounidense hacia América Latina en Rodríguez, 2012, pp. 277-309. En el interior del Departamento de Estado, la estructura administrativa más directamente implicada en la supervisión del FLP fue el United States Information and Educational Exchange Program (USIE).

10 Significativamente, en un informe preliminar sobre España (Country Plan) elaborado por el United States Information \& Educational Exchange Program (USIE) en 1951, en el último eslabón de los targets groups se encontraban las "clases rurales", que no suscitaban ninguna valoración particular. DELGADo, 2012, pp. 235-276.

11 En este proyecto también se implicaron organizaciones de la sociedad civil norteamericana (muy cercanas a la administración) como la Ford Foundation. Esta fundación colaboró estrechamente -durante las décadas de 1960 y 1970- con el Banco Urquijo, a través del Programa de seminarios organizado por su Sociedad de Estudios y Publicaciones (SEP) alguno de ellos dedicados en exclusiva a cuestiones agrarias (1966-1968). La orientación que se dio a los problemas relacionados con la transformación de la agricultura, se conciliaba bien con los objetivos que, en este ámbito, pretendían alcanzar los sucesivos Planes de Desarrollo. Véase De Santiesteban, 2009, pp. 159-191, y en especial pp. 176-187. 
Otro asunto de interés tiene que ver con el papel que el mundo rural jugó como potencial receptor de la propaganda norteamericana en España entre 1945 y el final de la década de $1950^{12}$. Las publicaciones periódicas, como Semanario Gráfico o Noticias de Actualidad, contaron con un número reducido de lectores entre las elites urbanas. Los documentales cinematográficos de producción norteamericana llegaron a sectores mucho más numerosos de la población, gracias a un sistema de unidades móviles (jeeps con proyectores) que permitían su proyección en ámbitos muy diver$\operatorname{sos}^{13}$. En 1954 los documentales dedicados a "Agricultura" (69) estuvieron entre los más numerosos, sólo por detrás de temáticas como "Industria y Ciencia" (80), "Panorama Americano" (75) o "Medicina" (72). En 1957, con 94 proyecciones, habían ascendido al segundo lugar del ranking, por detrás de los dedicados a "Medicina e Higiene" (98). Cabría explorar a qué objetivos respondieron estas realizaciones de temática agraria, en qué medida la población rural tuvo acceso a dicho material y cuál fue la impronta del mismo.

En septiembre de 1953 España y los EE.UU. firmaron un Convenio de Ayuda Económica ${ }^{14}$. Con su formalización, una misión económica especial norteamericana se estableció en España con el encargo de supervisar su correcto desarrollo. Esta fue la principal vía de acceso del sector agrario español a la asistencia técnica norteamericana.

De modo más genérico, la ayuda americana fue relevante para el sector agrario español, al permitir la compra, a través de los créditos del Eximbank y del Development Loan Fund, de maquinaria y fertilizantes químicos, así como la importación de excedentes agrarios (cereales, algodón o aceite de soja) procedentes de los EE.UU. ${ }^{15}$. El valor monetario de la asistencia técnica norteamericana a la agricultura española ha sido cuantificado en 5.500 millones de pesetas entre 1953 y $1964^{16}$.

La reorientación del conjunto de la política económica del régimen, iniciada con el Plan de Estabilización de 1959, no se debió solamente a las limitaciones de la estrategia autárquica. Ciertamente, la escalada de la inflación y el inminente colapso de la balanza de pagos hacían imperativa la consecución de la estabilidad macroeconómica, condición sine qua non para una posterior integración en diversos organismos internacionales y europeos, de ahí que las recomendaciones de los mismos (en particular del FMI) fuesen tenidas muy en cuenta a la hora de diseñar tanto la política

12 LEÓN, 2009, pp. 133-158, y en especial: pp. 139-140; 146-147 y 157. También LEÓN, 2012, pp. 197-234.

13 Las competencias sobre la divulgación de propaganda cinematográfica norteamericana en España eran del United States Information Service (USIS).

14 Para la caracterización global del Convenio de Ayuda Económica, seguimos a ViñAs, 2003, pp. 269-285, y más en particular, pp. 276-277 y 283-284. El Convenio de Ayuda Económica tenía una vigencia inicialmente limitada, unos tres años, hasta el 30 de junio de 1956.

15 Una parte de la ayuda económica norteamericana resultó fundamental durante los años cincuenta para el abastecimiento de la población española. La Public Law (PL) 480 permitía la venta a España de excedentes agrícolas a pagar en moneda local (pesetas) lo que no comprometía las escasas reservas de divisas españolas. También posibilitaba el intercambio de estos excedentes por materias primas estratégicas e incluso la canalización de donaciones a través de organizaciones caritativas, como las que desde 1954 realizó, a través de Cáritas, la National Catholic Welfare Conference. Viñas, 2003, pp. 288-291. MíngueZ, 1997, pp. 421-462.

16 Barciela, 2011, p. 213; Calvo, 2001, pp. 253-275. 
económica en general como las políticas sectoriales ${ }^{17}$. Pero además, la extensión de la sociedad de consumo, a tenor de lo que parecía suceder en otros estados, contribuía a la disminución de la conflictividad social y favorecía la aceptación del sistema capitalista ${ }^{18}$.

Con todo, y en el marco de las teorías político-ideológicas de modernización económica predominantes en aquel periodo, los organismos internacionales detectaban a comienzos de la década de los sesenta la persistencia de serios problemas de funcionamiento interno en la agricultura española. En 1962 se publicaba el Informe del Banco Mundial sobre la situación económica de España, que dedica su Sección IV al sector agrario ${ }^{19}$. Se contemplaba la necesidad de adaptar la producción a la demanda prevista en años venideros y se recogían toda una serie de medidas para mejorar la eficiencia productiva, desde los sistemas de regadío (donde la inversión resultase rentable), la reforma de la estructura territorial (a través del incremento de los trabajos de concentración parcelaria, la reforma de la legislación de arrendamientos o el aprovechamiento privado de las propiedades comunales), la conservación de los suelos, la mejora de semillas y ganado, el uso adecuado de abonos y maquinaria y la mejora de la investigación técnica, los servicios de extensión agrícola y el sistema educativo. Se recomendaba asimismo la reducción del intervencionismo estatal en la economía.

El Informe se acogía con reservas, lo que apunta a la existencia de posicionamientos diferentes entre las élites del régimen, que cabría estudiar más a fondo. Así, la intervención estatal seguía teniendo sentido para algunos autores, que en ocasiones continuaban recurriendo a la asociación retórica del campo con la esencia de la patria para justificar aquélla ${ }^{20}$.

En 1965 se realizaba otro informe, éste orientado de manera específica al sector agrario, promovido por el BIRD (Banco Internacional de Reconstrucción y Desarrollo) y la $\mathrm{FAO}^{21}$. De nuevo, se recogían orientaciones para una agricultura más racional y eficiente, con el horizonte de la década siguiente en perspectiva. Posteriormente a la realización de ambos informes, se implementaron una serie de préstamos a cofinanciar entre el BIRD y el gobierno del régimen ${ }^{22}$.

A continuación nos centraremos en el ámbito concreto del extensionismo agrario y la influencia del modelo norteamericano en la articulación del Servicio de Extensión Agraria en España (1955).

Para el ámbito europeo, fue la propia OECE, creada con el objetivo de coordinar la distribución de la ayuda americana del Plan Marshall, la que canalizó la influencia del modelo extensionista norteamericano. En la década de 1950, tanto en la Agencia para el Desarrollo Internacional (Agency for International Development, AID) como

17 Barciela - López Ortiz, 2003, pp. 55-94. Sobre el Plan de Estabilización y el Bienio Pre-estabilizador: Carreras - Tafunell, 2004, pp. 322-329; Comín, 2002; Comín - HernándeZ - Llopis, 2002, pp. $364-376$.

18 Ramos - Pires Jiménez, 2009, pp. 36-37; Molinero - Ysás, 2008, p. 60; Cazorla, 2010, pp. 149-155; Hernández Burgos, 2013, pp. 286-299.

19 Informe del Banco Mundial (1962), Oficina de Coordinación y Programación Económica.

20 Lamo de Espinosa, 1962, pp. 11-54; Zorrilla Dorronsoro, 1962, pp. 61-78.

21 El desarrollo de la agricultura en España (1966), Informe del Banco Internacional de Reconstrucción y Fomento y de la Organización de las Naciones Unidas para la Agricultura y la Alimentación, Ministerio de Hacienda.

22 Hernando, 2001, pp. 43-63. 
en la misma Organización para la Alimentación y la Agricultura (FAO), ya trabajaban expertos en extensión para prestar asistencia en este terreno ${ }^{23}$.

\section{LA INFLUENCIA DEL MODELO NORTEAMERICANO EN EL EXTENSIONISMO AGRARIO EN ESPAÑA (1955-1975)}

La influencia de la filosofía y práctica de la extensión de acuerdo con los parámetros dominantes en los EE.UU., tanto en Europa como en América Latina, se vehiculó a través de dos canales principales: el asesoramiento directo por parte de expertos que se desplazan a los países afectados y la formación de personal de esos países o bien en los EE.UU., o en otros lugares con una extensión especialmente desarrollada y en sintonía con el modelo norteamericano, como el Centro Agrícola Internacional de Wageningen, en los Países Bajos ${ }^{24}$. Estas mismas estrategias eran las seguidas en el Programa de Asistencia Técnica que incluía el Plan Marshall para los países bajo su influencia ${ }^{25}$.

El principal estudio para acercarse a la influencia del modelo norteamericano en el Servicio de Extensión Agraria en España, al igual que la principal monografía de conjunto sobre el SEA, es la realizada por Sánchez de Puerta, a la que acudiremos como referente fundamental para acercarnos a esta cuestión ${ }^{26}$.

España no formaba parte de los Estados europeos incorporados al Plan Marshall, pero, como hemos mencionado, se articularon mecanismos de ayuda económica alternativos para el país. Para valorar las posibles aplicaciones de esa ayuda, se organizó una Misión Económica por parte de los EE.UU. El director de la misma, Charles Fossum, encargó un estudio sobre los instrumentos de información agrícola en España, para valorar la posibilidad de organizar un servicio consultivo a nivel estatal. Recibido el informe, elaborado por Layne R. Beaty, un experto en información agrícola de la Office of the Special Representative to Europe (OSR), con sede en París, Fossum contactó con el Ministro de Agricultura (Rafael Cavestany), el Presidente de las Estaciones Experimentales (Miguel Echegaray) y el Director General de Coordinación, Crédito y Capacitación (Santiago Pardo Canalís). El entonces Ministro de Agricultura fue además invitado por el director de la Misión a viajar a los EE.UU. para conocer el trabajo del Servicio de Extensión Cooperativo norteamericano. Fue al regresar de ese viaje cuando desde el Ministerio se publicó la Orden por la que se creaba el Servicio de Extensión Agrícola ${ }^{27}$. Seguidamente, dos técnicos del Ministerio fueron enviados de nuevo a EE.UU. durante una estancia de seis meses para conocer el funcionamiento del Servicio. A su vez, de acuerdo a la estrategia formativa

23 SÁnchez De Puerta, 1996, p. 138.

24 Ibídem, p. 136. Así sucedía, por ejemplo, en el caso de Portugal, donde al amparo del "Technical Assistance \& Productivity Programme" del Plan Marshall se desarrollaron 25 proyectos agrícolas entre 1951 y 1956, en muchos de los cuales se puede rastrear la impronta de la filosofía extensionista norteamericana: Rollo, 2011, pp.107 - 133.

25 Moya, 2011, pp. 13-25; PuIG, 2003, pp. 109-129.

26 SÁnchez De Puerta, 1997, pp. 913-948. Seguimos aquí también a Fernández Prieto, 2007, pp. 331-344.

27 Orden Ministerial de 15 de septiembre de 1955 por la que se encomienda a la Dirección General de Coordinación, Crédito y Capacitación Agraria el Servicio de Extensión Agrícola (BOE, 273, 30/09/1955). 
antes mencionada, dos asesores norteamericanos (Emilio Tejada y Anacleto G. Apodaca) fueron enviados a España para formar a los primeros agentes ${ }^{28}$.

El manual de Extensión "Enseñando al agricultor" (1954), de Anacleto G. Apodaca, fue referencia principal en los primeros años del SEA. En esta obra se seguían los principios del extensionismo agrario norteamericano, coincidiendo en buena medida con los "Principios de Extensión Rural" de Penders. Para este último autor -junto con Mosher, nombres principales en la teoría extensionista norteamericana de esas décadas-, el trabajo con la mujer y la juventud rurales era una estrategia necesaria en la actividad de la extensión agraria ${ }^{29}$. El propio Anacleto G. Apodaca realizó en 1959 un audiovisual sobre el Servicio de Extensión Agrícola. Aunque se hacía referencia al trabajo con mujeres y niños, la mayor parte del metraje estaba centrado en el papel del SEA en el ámbito productivo ${ }^{30}$.

Según escribía, al año siguiente (1960), Gómez Ayau, ingeniero agrónomo y economista, y por entonces Director General del SEA (hasta que en 1962 fue relevado por García Gutiérrez) a finales de la década de los cincuenta, la atención del Servicio de Extensión norteamericano no sólo se dirigía a la mejora de la producción, sino también a la comercialización de la misma, la gestión de las explotaciones y el interés por el potencial de los "dirigentes" locales. Más aún, el trabajo con los jóvenes, la familia y el conjunto de la comunidad local, eran los aspectos que marcaban una diferencia en positivo respecto de los Servicios de Divulgación que trabajaban en muchos Estados europeos, e incluso respecto del propio Servicio norteamericano en sus inicios, también entonces centrado en la divulgación técnica ${ }^{31}$. Sin embargo, como veremos más adelante, durante los primeros años del SEA su trabajo se centró en la difusión técnica, y no será hasta el cambio de década cuando sus programas y actividades adquieran un perfil más social. Se destaca además una de las principales ideas-fuerza de los agentes del SEA: el trabajo con el agricultor o, de otra manera, la necesidad de su papel activo en el cambio ${ }^{32}$.

28 Rafael Cavestany fue ministro de Agricultura de 1951 a 1957. Su sucesor en el cargo fue Cirilo Cánovas, que continuó las líneas establecidas por su predecesor. Sánchez De Puerta, 1996, pp. 392-395.

29 Ibídem, pp. 148-149, 406. Según indica este autor, Apodaca nació en Texas y se licenció como ingeniero agrícola en Nuevo México, donde entró a formar parte del Servicio de Extensión Cooperativo norteamericano. Posteriormente obtuvo el doctorado en Extensión Agrícola por la Universidad de Cornell y marchó a Honduras para enseñar esta disciplina. Fue allí donde editó, en 1954, el mentado manual (p. 406).

30 GARCíA APODACA, 1959a (Documental): http://ruralmedia.eu/2009/05/la-extension-agricola-en-espana/ En este documento audiovisual el propio origen del SEA se vincula directamente a su papel en la transmisión a los agricultores de nuevos métodos orientados al aumento de la productividad, que se ensayan en centros de investigación agronómica. También se muestran algunos ejemplos, como la promoción del uso de insecticidas para combatir plagas y de herbicidas contra las «malas hierbas», el uso de semillas seleccionadas, los nuevos métodos de ensilaje, la orientación en la poda «racional» de los árboles, los consejos para tomar muestras de suelos que permitan abonar correctamente, el complemento del abono natural con el químico o el fomento de medidas para la conservación de suelos y (vinculado a ésta) la repoblación forestal.

31 Gomez Ayau, 1960, pp. 53-56.

32 Ibídem, pp. 35-36. Gómez Ayau escribe este artículo poco después de regresar de un viaje a EE.UU. y Canadá (otoño de 1959) en el que formó parte de una Misión de Directores de Agricultura y Directores de Servicios de Vulgarización Agrícola de la Agencia Europea de Productividad, un organismo en la órbita de la OECE. Durante el viaje visitó las Universidades de Tucson (Arizona) y Purdue (Indiana), así como los Servicios de Extensión Agraria que se apoyaban en estos dos centros. Asistió también a la Convención anual de Land Grant Colleges (universidades agrícolas). 
Una valoración similar la encontramos en Sanders et al. (1966). Para estos autores, además, a mediados de los sesenta, los objetivos específicos del Servicio de Extensión norteamericano eran coherentes con los generales de los EE.UU.: garantizar la abundancia de alimentos, la familia y el hogar como núcleo social básico y fomentar el desarrollo del liderazgo ${ }^{33}$.

Para entender la organización del SEA necesitamos, por tanto, situarlo adecuadamente en el contexto político e ideológico internacional del momento, pero también en el de una política agraria estatal que buscaba proporcionar los marcos que favoreciesen la necesaria modernización de la agricultura española. Por esas mismas fechas se promulgaban las Leyes de Concentración Parcelaria (1952 y 1955) y la Ley de Fincas Manifiestamente Mejorables (1953) además de consolidarse la política de colonización y repoblación forestal ${ }^{34}$.

Los años cincuenta marcaron una etapa de cambio en la política agraria española, dado que fue entonces cuando la orientación tecnocrática y liberalizadora fue ganando peso respecto de los principios nacionalsindicalistas. Se renunciaba al ideal autárquico para iniciar una reforma del sector agrario que consiguiese unos mayores niveles de productividad y eficiencia. Buena parte de las políticas de esta etapa, sin embargo, habían sido ya diseñadas en la década anterior. Más aún, algunas de las principales hundían sus raíces en proyectos previos, ya formulados durante el gobierno republicano (1931-1936) o incluso en períodos anteriores. El estudio de las políticas diseñadas en etapas previas, por tanto, nos invita a la reflexión respecto de las diferentes concepciones de modernidad y los proyectos de modernización consecuentes que pueden promocionarse desde las esferas de poder. En el caso del régimen franquista, podemos detectar el paso de un inicial modelo autoritario de modernización, en el que tenían una fuerte presencia los principios fascistas, hacia otro de perfil tecnocrático, en el contexto de la hegemonía de la Teoría de la Modernización ${ }^{35}$.

La creación del SEA supuso un cambio en el aspecto relacional entre técnicos y sociedad rural, debido al predominio de técnicos medios y la metodología de trabajo empleada ${ }^{36}$. En este ámbito específico de la Extensión, la influencia del modelo estadounidense se concretó en la formación de los primeros agentes a través de la preparación de los mismos por parte de los asesores Tejada y Apodaca. En base a un acuerdo entre el INIA (Instituto Nacional de Investigaciones Agronómicas) y el IIA (Institute of International Education), una Fundación filantrópica norteamericana, se reconstruyó un convento situado en Jerez de la Frontera (Cádiz), en el que se

33 SANDERS, 1966, pp. 130-131.

34 Ley de 20 de diciembre de 1952 sobre Concentración Parcelaria (BOE, 23/12/1952); Ley de 20 de julio de 1955, por la que se complementa la de 20 de diciembre de 1952 sobre Concentración Parcelaria (BOE, 21/07/1955; Ley de 3 de diciembre de 1953 sobre declaración de fincas manifiestamente mejorables, BOE 04/12/1953).

35 Cabana - Díaz-Geada, 2014; Fernández Prieto - Pan Montojo - Cabo, 2014, pp. 19-36.

36 Cabana - Díaz-Geada, 2010. La rivalidad entre cuerpos técnicos o, en otros términos, la posición subordinada de los técnicos medios respecto de los ingenieros, está también en relación con el importante grado de autonomía del SEA y su trabajo en base a la labor de agentes polivalentes. FERnÁNDEZ PRIETO, 2007, pp. 341-342; Gómez - Luque, 2007b. El Servicio de Extensión Cooperativo norteamericano, sin embargo, estaba compuesto por agentes y especialistas en distintas ramas agrarias que asesoraban a los primeros. SÁnchez De Puerta, 1996, p. 400. 
dio inicio a dicha formación. En esta misma provincia se establecieron las primeras agencias-piloto $^{37}$. El INIA cedió asimismo la finca experimental de "El Encín", en Alcalá de Henares (Madrid) para albergar la realización del primer ciclo formativo de los agentes. En régimen de internado, esta formación se centraba en la Teoría de la Extensión, unas nociones básicas de Sociología y Psicología y conocimiento general sobre Agricultura. Posteriormente, los agentes se trasladaban a las agencias, que iban creciendo en número y donde también seguían contando con la orientación de los asesores norteamericanos ${ }^{38}$. Además de la orientación directa por parte de asesores norteamericanos en España, siguiendo la estrategia general que comentamos anteriormente, también los que serían cargos directivos del SEA en sus años de organización, recibieron formación metodológica en los EE.UU. o en Holanda, en el Centro formativo de Wageningen, asistiendo al Curso Internacional allí realizado. Así, en 1959 y 1960 varios agentes e inspectores participaron en cursos especializados sobre diversos temas, caso de estrategias de información o trabajo con los jóvenes.

Con todo, como anota este autor, además de la participación en estos cursos, para valorar la impronta de los participantes en su justa medida, cabría estudiar el nivel de aprovechamiento ${ }^{39}$.

Durante los primeros años del SEA, tal y como se especifica en la Orden de creación, y de acuerdo a los criterios establecidos en el acuerdo firmado con el gobierno de los EE.UU., el trabajo de los agentes se centra en la difusión técnica, con el aumento de la productividad agrícola (entendido además como vía de mejora del nivel de vida en el campo) como principal objetivo ${ }^{40}$.

Inicialmente, la labor del SEA se desarrolló en zonas de colonización y concentración parcelaria, para extenderse posteriormente al conjunto de la población rural. Si durante su primer quinquenio el ámbito productivo copaba su actividad, con el cambio de década empezó a prestarse mayor atención a la juventud y a la mujer. Según indican Sánchez de Puerta, Gómez Benito y Luque Pulgar, la década de los sesenta marcó un cambio respecto de la anterior, con la adopción de nuevos principios teóricos y metodológicos, en un momento de expansión del Servicio. Para estos últimos autores, el trabajo de proximidad propio del SEA derivó en un cambio de enfoque

37 Sánchez de Puerta explica que esta localización se debe a la procedencia de los fondos económicos para implementar el acuerdo, que procedían de la herencia de Elías Aguja, español emigrado a Nueva York, para la creación de una "Sociedad de Buenos Samaritanos" para "ayudar a los pobres de Cádiz". La Sociedad administradora consideró oportuna la promoción de alguna iniciativa educativa y lo consultó con el Instituto Internacional de Educación, que a través de Earnest Maes, en contacto con Fossum, apoyó la organización del SEA. Sánchez De Puerta, 1996, p. 395. También García Apodaca, 1958, pp. 7-8.

38 SÁnchez De Puerta, 1996, p. 395; Rodríguez Troncoso, 2009, pp. 20-22.

39 Así, el asesor Apodaca valoraba estas iniciativas formativas de manera positiva, aunque señalaba que el conocimiento de la lengua inglesa debería mejorarse para sacar mayor partido a las estancias. GARCía Apodaca, 1959a, p. 19; SÁnchez De Puerta, 1996, p. 396.

40 Orden Ministerial de 15 de septiembre de 1955 por la que se encomienda a la Dirección General de Coordinación, Crédito y Capacitación Agraria el Servicio de Extensión Agrícola (BOE, 273, 30/09/1955); SÁnchez De Puerta, 1996, p. 397. El trabajo al principio, se orientó a mejorar los rendimientos de los cultivos tradicionales, maíz, patatas, praderas y en los ganaderos, el vacuno que era el dominante. Paralelamente [...] iniciamos campañas para incentivar a los propietarios a solicitar la concentración parcelaria. MARTín GARCÍA, 2012, pp. 127-128. 
propio que tuvo su elemento esencial en la concepción integral de la extensión ${ }^{41}$. Así, desde un punto de vista teórico se transitaría desde la mera transferencia de tecnología, de arriba a abajo, a una estrategia más social y cultural, abordando el cambio desde la base. En esta dirección se crea la Sección de Desarrollo de Comunidades (1966), que sumada a la labor de las agentes de Economía Doméstica (1960) y el trabajo con los planteles de jóvenes (1962), conforman las "cuatro patas" del trabajo del SEA. Además de cuestiones técnicas, por tanto, los agentes organizaban proyectos diversos con los jóvenes de los planteles y las agentes de Economía Doméstica diseñaban actividades varias con las mujeres (desde un curso de cocina hasta la organización de una cooperativa) ${ }^{42}$. La creación de los SEGEs (Seminarios de Gestión de Explotaciones) en 1967, es una muestra más de esta reorientación social del Servicio, dada la importancia atribuida en los mismos a los aspectos sociales y culturales. En cuanto al Desarrollo Comunitario, su origen tiene que ver con la dificultad práctica que encontraron los agentes para la organización de cooperativas en sus áreas de trabajo y, por el contrario, la colaboración vecinal que detectaban a la hora de solucionar problemas que afectaban al colectivo. De ahí la promoción de iniciativas comunitarias que facilitasen la mejora de ciertos servicios (caminos, alumbrado público, construcciones de uso común) al tiempo que reforzaban la experiencia del trabajo en colectivo $^{43}$.

El cambio de orientación coincide con el de la cabeza dirigente del Servicio. García Gutiérrez, nuevo Director General, recibió también formación en EE.UU. y conocía la manera de trabajar del Servicio hasta su llegada, basada en el modelo norteamericano. Su procedencia familiar y su experiencia de trabajo, sin embargo, influyeron notablemente en su manera de entender la extensión, condensada en el lema "Extensión no trabaja con programas, trabaja con problemas" ${ }^{44}$. La primacía de los problemas sociales respecto de los técnicos era compartida por Salvador Chico, director de la Sección de Desarrollo Comunitario y seguidor de los postulados educativos de Paulo Freire, al que se debe la introducción de los sociólogos en las agencias ${ }^{45}$.

Durante la etapa directiva de García Gutiérrez, se promovió la descentralización del Servicio, su autonomía respecto de otras instituciones relacionadas con la agricultura, la prioridad del nivel local sobre el regional, el trabajo de proximidad con los agricultores y la formación y perfil social (y no meramente técnico) de los agentes ${ }^{46}$. La estrategia extensionista promovida por García Gutiérrez no aparece sistematizada en ningún escrito, pero sus consejos fueron requeridos por los gobiernos de otros

41 Gómez - Luque, 2007a, pp. 24-25. Sobre la hegemonía del paradigma de Transferencia De Tecnología (TDT) y sus alternativas en la esfera del extensionismo agrario. SÁnchez De Puerta, 1996, pp. 248-318.

42 En el caso del trabajo con jóvenes y mujeres, el SEA hubo de negociar con el Frente de Juventudes y la Sección Femenina de Falange, dado el choque de competencias. En EE.UU. los jóvenes se organizaban en los llamados "Clubs 4-H", pero dado que tal término podía ser traducido como "asociación" y su creación estaba prohibida por ley, se optó por la denominación de "planteles". Con todo, en España, anteriores a los Planteles fueron las Academias de Extensión, en las que se impartía formación a los hijos de los agricultores que habían abandonado sus estudios. SÁnchez De Puerta, 1996, pp. 420, 423.

43 Ibídem, 1996, p. 411.

44 Entrevista a García Gutiérrez en SÁnchez De Puerta, 1996, p. 423.

45 Ibídem, p. 412; Díaz-GEADA - LANERo TÁBOAS, 2012.

46 SÁnchez De Puerta, 1996, pp. 413-414. 
estados y la propia $\mathrm{FAO}^{47}$. En 1971, el SEA alcanzaba su mayor grado de expansión territorial, con 755 agencias comarcales distribuidas por todo el Estado.

Cuadro 1. Evolución del número de Agencias Comarcales del SEA.

\begin{tabular}{|c|c|}
\hline AÑO & Agencias Comarcales del SEA \\
\hline 1957 & 44 \\
\hline 1959 & 85 \\
\hline 1961 & 135 \\
\hline 1965 & 364 \\
\hline 1967 & 500 \\
\hline 1969 & 615 \\
\hline 1971 & 755 \\
\hline
\end{tabular}

Fuente: SÁnchez De Puerta, 1996, pp. 396 y 421.

El abordaje colectivo de diferentes problemas con las comunidades locales puso de manifiesto el carácter complejo y paradójico de la implementación de determinadas políticas agrarias guiadas por modelos teóricos específicos en conflicto y negociación con unas comunidades rurales que interactuaban de acuerdo a otras cosmovisiones e identidades ${ }^{48}$. El estudio detenido de las condiciones locales, así como el recurso a métodos de trabajo participativos, convivió con una vocación transformadora en favor de la consecución de unas determinadas condiciones de bienestar en el ámbito rural, en el que de acuerdo a los requerimientos que el sistema de mercado demandaba en ese momento, resultaba cada vez más difícil permanecer desarrollando una actividad agraria.

Otra muestra de las diferentes construcciones y proyecciones que pueden derivarse del uso de metodologías específicas, así como del carácter complejo de los procesos con los que tratamos, está en la experiencia de una minoría de agentes que fueron más allá de las problemáticas concretas para cuestionarse el marco político y económico en que tales situaciones adquirían sentido. Así, unos pocos unieron su trabajo por un rural con mayor bienestar a la lucha por la democracia.

A la hora de abordar el estudio del Servicio de Extensión Agraria, por tanto, resulta necesario atender a la evolución del marco político y social estatal en el que se desarrolló, así como a los universos locales concretos en que los proyectos modernizado-

47 Ibídem, pp. 415-422; García GutiérRez, 1994, pp. 167-173.

48 Díaz-Geada, 2013, pp. 122-154. 
res tornan en realidades de cambio más o menos resistido o negociado, pero también es preciso, como tratamos de mostrar en este texto, atender al marco ideológico y de relaciones internacionales en que se explica la organización de numerosos servicios de extensión a lo largo y ancho del globo.

\section{EL SERVICIO DE EXTENSIÓN AGRARIA (SEA) ESPAÑOL EN EL MARCO DEL EXTENSIONISMO INTERNACIONAL DE POSGUERRA}

Dedicaremos el último apartado de nuestro texto a la contextualización del Servicio de Extensión Agraria (SEA) español en el ámbito internacional. El estudio comparado de distintos servicios de extensión permite comprender con mayor amplitud su sentido y el papel jugado por la ayuda técnica norteamericana. En esta ocasión, priorizaremos la comparación entre el extensionismo agrario de matriz norteamericana en España y América Latina durante la Guerra Fría.

En realidad, el paradigma modernizador diseñado en las décadas de 1950 y 1960 para las agriculturas de países "en vías de desarrollo" del Sudeste asiático o de América Latina, podía perfectamente servir para los países "más atrasados" de la Europa del Sur (España, Portugal o Grecia). Se podría ir más allá y afirmar incluso que la influencia teórica del paradigma modernizador norteamericano de posguerra fue tan potente que su onda expansiva alcanzó contextos geográficos y políticos muy diversos, así como a agriculturas "nacionales" con posiciones relativas muy diferentes respecto de lo que la ortodoxia modernizadora consideraba la evolución normativa recomendable para el sector agrario dentro del conjunto de la economía de cualquier Estado $^{49}$. El incremento de la productividad agraria mediante la transferencia de tecnología a gran escala fue un objetivo asumido y compartido por las elites políticas españolas, filipinas, mexicanas, brasileñas o costarricenses, pero también por sus homólogos de los Países Bajos, Francia o por los planes del Departamento de Agricultura para la transformación agrícola de los estados sureños en los EE.UU. ${ }^{50}$.

A mediados de la década de 1960, una monografía colectiva sobre el Cooperative Extension Service de los EE.UU. valoraba la situación de los Servicios de Extensión Agraria en diferentes países del bloque capitalista ${ }^{51}$. Con una óptica probablemente condicionada por los presupuestos de partida de la obra, se trazaba un cuadro general en el que el Servicio norteamericano se convertía en la medida hacia la que todos los demás tendrían que evolucionar. Así, se consideraba que los países escandinavos, Canadá, Australia y Japón habían implementado Servicios nacionales de Extensión Agraria muy semejantes al norteamericano. Otros Estados europeos, como el Reino Unido o los Países Bajos -que habían desarrollado un importante entramado institu-

\footnotetext{
49 Hemos reflexionado en profundidad a este respecto en Díaz-Geada - Lanero Táboas - FernándezPRIETO, 2012.

50 Sobre la Extensión Agraria en los Países Bajos, véase Karel, 2006, 2010. Para Francia véase Brunier, 2012. Algunas referencias al modelo de los Centres d'Etudes Techniques Agricoles (CETA) franceses en Gómez Ayau, 1960, pp. 34-37. Para el caso brasileño, Da SiLva, 2014.

51 Maunder, 1966, pp. 7-12. Citado y reproducido parcialmente en Sánchez De Puerta, 1996, pp. 132135 .
} 
cional de la innovación al menos desde mediados del siglo XIX- ofrecían, según la obra, un modelo de servicio en el que predominaban aún los aspectos "tradicionales" (consultivos) directamente vinculados a los problemas de la producción agrícola y, por ello, necesitaban de un cambio de orientación que ensanchase su ámbito de actuación. En cambio, en sus antiguas colonias habían ensayado modelos de extensionismo que ponían mayor énfasis en el desarrollo comunitario. Precisamente en India y Pakistán, dos "nuevos Estados" que habían obtenido su independencia del imperio británico en 1945, estos autores identificaban programas de desarrollo comunitario vinculados a acciones sociales de carácter local, pero que, en su opinión, deberían reorientarse hacia un modelo de extensión ligado a las instituciones gubernamentales con responsabilidades sobre el sector agrario y a las universidades, así como profundizar en el desarrollo de la formación técnica. En síntesis, copiar o imitar algunos de los rasgos característicos del extensionismo norteamericano ${ }^{52}$.

Finalmente, en América Latina, África y Oriente Medio, la necesidad de establecer programas de extensión en la estela del modelo norteamericano sería, para los citados autores, acuciante. En estas regiones del planeta el desarrollo material del mundo rural facilitaría la construcción de parapetos sociales, económicos e ideológicos contra la posible expansión de propuestas alternativas al capitalismo.

Retomando el caso del SEA español, los paralelismos entre éste y los "Servicios Nacionales" impulsados por los EE.UU. en varios países latinoamericanos después de la Segunda Guerra Mundial son notables. La diferencia fundamental estaría en su origen más tardío y en las motivaciones geoestratégicas de los EE.UU. en cada contexto. La cooperación en materia agraria con la dictadura de Franco había sido inexistente en la década de 1940. En cambio, los países "amigos" de Centro y Sudamérica, se convirtieron en el curso de la Segunda Guerra mundial en un objetivo prioritario para la política exterior norteamericana, preocupada por la construcción de alianzas que garantizasen el aprovisionamiento alimentario para sus tropas y la disponibilidad de materias primas (caucho, quinina, metales estratégicos...) fundamentales en una coyuntura de esfuerzo bélico. Estos "Servicios Nacionales" darían pues continuidad a las actividades de cooperación y al tejido institucional previamente creado, incorporando a sus objetivos, en el marco de la Guerra Fría, los principios de la contención geopolítica del comunismo, asunto que, a la altura de los años cincuenta, no inquietaba a la administración norteamericana en el caso de España.

Las vías de implantación y la forma de organización de estos "Servicios Nacionales" guardan semejanzas significativas con el caso del SEA. Aunque las actividades de aquéllos desbordaban el ámbito de la extensión agraria, lo relacionado con ésta parece haber sido el aspecto más relevante de sus funciones. Dicho de otro modo, el

52 Sobre los programas e instituciones británicos en el ámbito de la "agricultura colonial", véase HodGE, 2010, 2007, en especial, pp. 207-253. Este autor, pp. 208-209, pone de manifiesto, en el caso del Imperio británico (pero también en las colonias francesas) la existencia de una "ofensiva por el desarrollo colonial" entre 1947 y 1951 a fin de servir a los intereses de la economía nacional británica, que implicaría un nivel de actuación estatal sin precedentes en ámbitos como el cambio agrario y el desarrollo rural. El extensionismo agrario jugaría un papel muy destacado en la implantación de diversos proyectos: conservación de suelos, producción de alimentos, mecanización del cultivo de arroz y algodón, reasentamiento de poblaciones locales, fomento de granjas colectivas, etc. 
extensionismo agrario se convirtió en la plataforma a partir de la cual desarrollar diversas acciones y programas de modernización agraria (proyectos de ingeniería civil, conservación de suelos, puesta en marcha de explotaciones modelo, establecimiento de parques de maquinaria agrícola, etc. $)^{53}$.

J. A. Fernández y W. Picado plantean la existencia de una dinámica de "clonación institucional" del Cooperative Extension Service de los EE.UU. que se repite en varios países latinoamericanos, hasta el punto de que en Costa Rica (1948), Honduras (1951) y Paraguay el nombre del organismo es idéntico: Servicio Técnico Interamericano de Ciencias Agrícolas (STICA), en Nicaragua (1953): Servicio Técnico Agrícola de Nicaragua (STAN), y muy semejante era también la denominación de su precedente peruano (1943): Servicio Cooperativo Interamericano de Producción de Alimentos (SCIPA) ${ }^{54}$. Estos "Servicios" eran el resultado de la colaboración entre diversos gobiernos latinoamericanos y el Instituto de Asuntos Interamericanos (IAIA) o, en otras ocasiones, la Oficina de Relaciones Agrícolas Exteriores de los EEUU. El caso español encaja en esa misma "lógica clonadora", aunque con casi una década de retraso con respecto de las primeras experiencias latinoamericanas, el tiempo que tardó el franquismo en ser reconocido por los EE.UU. y la comunidad internacional.

El sistema de cofinanciación paritaria de las estructuras institucionales y de las actividades de los "Servicios [agrícolas] Nacionales" entre los EE.UU. y los gobiernos de los países latinoamericanos en los que estos se establecieron, está próximo a las fórmulas que sirvieron para sufragar los diferentes programas de colaboración de la administración norteamericana en la España de los cincuenta. También lo hace el control que inicialmente (a través de los recursos económicos y de los técnicos responsables de la dirección y de la formación del personal local) los EE.UU. mantuvieron sobre los STICA americanos, o el SEA español ${ }^{55}$. Si, por ejemplo, los directores del STICA costarricense fueron hasta 1951 estadounidenses, en el caso español, un experto norteamericano, Anacleto G. Apodaca, a quien se había encomendado poner en marcha (en 1951) un Servicio de Extensión Agraria en Honduras, fue uno de los encargados, como ya explicamos, del montaje y puesta en funcionamiento del SEA en $1955^{56}$.

La dimensión "regional" (y geoestratégica) del asesoramiento técnico de los EEUU a las agriculturas latinoamericanas, se hace también evidente si atendemos a la puesta en marcha de iniciativas educativo-formativas que sobrepasan la escala estatal. En Honduras, en 1943, con la financiación de la United Fruit Company se creaba la Escuela Agrícola Panamericana (más conocida como El Zamorano por el nombre de la finca en que se instaló). En ella se formaban cada año, con una orientación eminentemente práctica, setenta jóvenes becados, procedentes "de cualquier

53 En 1953, los Servicios de Extensión recibían en Perú 300.000 \$ USA, el 50\% del presupuesto total del SCIPA para ese año. Citado en Mosher, 1957, p. 49.

54 FernándeZ - PicAdo, 2013.

55 Acerca de la historia del Servicio Técnico Interamericano de Ciencias Agrícolas (STICA) de Costa Rica, véase PICADO, 2012a, pp. 185-208.

56 El STICA hondureño contaba a finales de 1952 con siete técnicos norteamericanos. En realidad, con el establecimiento del Servicio en Honduras se aspiraba a crear un verdadero Ministerio de Agricultura con su servicio de Extensión Agraria, estructuras institucionales de las que carecía este país a comienzos de la década de 1950. Véase García ApodacA, 1953, p. 26. 
República de habla hispana" ${ }^{57}$. Numerosos agentes de extensión agraria y técnicos agrarios de nivel superior ("especialistas"), que engrosarían las filas de los STICA y los servicios ministeriales de numerosos estados latinoamericanos realizaron sus estudios en El Zamorano.

Por su parte, la Organización de Estados Americanos (OEA) estableció en Turrialba (Costa Rica), el Instituto Interamericano de Ciencias Agrícolas (IICA), donde se impartían cursos intensivos sobre extensión agraria y otros de especialización alrededor de cultivos como el café, el cacao o la caña de azúcar. En relación con los objetivos que nos planteamos en este trabajo, es interesante hacer notar que el IICA prestaba desde comienzos de la década de 1950 una creciente atención "al estudio Económico-Social de los problemas de los países Latino Americanos", incorporando "ciencias que tratan de los aspectos sociológicos del pueblo" 58 . Una comparación entre el funcionamiento interno, los programas académicos y el perfil sociológico de los estudiantes en estas instituciones y en el Centro Agrícola Internacional de Wageningen sería del máximo interés y podría, tal vez, aproximarnos a algunos elementos comunes del paradigma de modernización agraria norteamericano en contextos socio-políticos muy diversos a priori.

Este control directo que los funcionarios norteamericanos ejercieron sobre los nuevos Servicios de Extensión plantea, a su vez, nuevos interrogantes: ¿Hasta cuándo duró en cada caso? ¿Cuándo y por qué se retiraron de la dirección y cómo se produjo el tránsito hacia una gestión "autóctona"? Cuestiones análogas deben formularse respecto de la evolución en el tiempo de los centros internacionales de formación agronómica.

Por otra parte, la comparación entre los casos latinoamericanos y el español permite aventurar alguna otra hipótesis. La situación peculiar (por su origen en parte externo) tanto de los STICA como del SEA dentro del organigrama de la administración agraria de estos países, parece encontrarse detrás del amplio grado de autonomía de que todas estas entidades disfrutaron en comparación con otras estructuras de la administración del Estado. En Costa Rica esa autonomía llevaría, desde 1950, a conflictos de intereses y competencias con servicios dependientes del Ministerio de Agricultura, así como a la definitiva integración de las actividades de extensión agraria en dicho Ministerio en 1956. En Perú, la capacidad del SCIPA para proyectarse dificultaría la emergencia, el reconocimiento público y, sobre todo, la "confianza" de los agricultores en otros servicios autóctonos creados por el Ministerio de Agricultura peruano para el desarrollo de una agricultura "eficiente" 59 . En el caso español, carecemos todavía de trabajos que expliquen cuáles (y cómo) fueron las relaciones entre el SEA y el Ministerio de Agricultura, así como entre los cuerpos técnicos al frente de cada uno de ellos (técnicos de nivel medio en el SEA e ingenieros agrónomos y forestales en el Ministerio) ${ }^{60}$. En cualquier caso, tanto los agentes de extensión del SEA como el personal con responsabilidades en la dirección del Servicio, han construido en torno a su pasado corporativo y a la historia del propio organismo una memoria

\footnotetext{
57 Ibídem, pp. 41-43.

58 Ibídem, pp. 46-47.

59 Mosher, 1957, p. 68.

60 Véase a este respecto Gómez BEnIto, 1996.
} 
que enfatiza el carácter autónomo de su actuación, así como la novedad y ruptura que el SEA habría representado respecto de las prácticas habituales de otras instancias agrarias de la dictadura, en especial en lo que hace a la relación que los agentes de extensión establecieron con las comunidades locales rurales. En cierto sentido, a través de esta memoria colectiva, el SEA viene a trascender al propio contexto histórico en que se fraguó la mayor parte de su actividad: el franquismo ${ }^{61}$.

Finalmente, la "misión" de modernización de la agricultura y de transformación de la sociedad rural como objetivos globales, su estructura organizativa, la metodología empleada (con su énfasis en la praxis demostrativa) y su trabajo con mujeres y jóvenes, aproximan a los STICA y al SEA, y remiten en todos los casos con bastante fidelidad a la matriz norteamericana de este modelo de extensionismo agrario ${ }^{62}$.

\section{CONCLUSIONES}

Tal como hemos expuesto en las páginas anteriores, la influencia del modelo de modernización promovido por los EE.UU. en muy diversos contextos geopolíticos durante la Guerra Fría, es un aspecto nodal a la hora de estudiar sus políticas agrarias. Si bien su funcionalidad política parece más evidente en diferentes países de América Latina, África o Asia, que los EE.UU. deseaban colocar bajo su órbita, no resulta sorprendente que sus postulados teóricos y las acciones políticas concretas que los acompañaban se pusieran también en práctica en dictaduras aliadas (como las de la Europa mediterránea) cuya evolución política era difícil de predecir una vez que sus dirigentes desaparecieran.

Si el reconocimiento político y la conversión de España en un aliado por parte de los EE.UU. fueron factores decisivos para la supervivencia del Régimen, el apoyo financiero y la asistencia técnica norteamericana realizaron una aportación significativa al cambio de rumbo que la política agraria franquista había iniciado a comienzos de la década de 1950.

Aunque no podemos medir su impacto concreto, el Servicio de Extensión Agraria fue sin duda una pieza relevante de un engranaje coherente de medidas políticas y legislativas encaminadas a la consecución de un ideal de agricultura "moderna" hegemónico en el conjunto del mundo "occidental" de la época.

La extensión del regadío hasta sus límites ambientales y tecnológicos, la reestructuración de las unidades productivas o la intensa capitalización de las explotaciones con inputs ajenos al sector (tractores y maquinaria agrícola en general, semillas y razas de ganado mejoradas genéticamente, fertilizantes y fitosanitarios, etc.) fueron procesos que se encuadraron en esta misma lógica. La política tecnocrática del fran-

61 Rodríguez Troncoso, 2009.

62 En Costa Rica el STICA creó los Clubes 4-S a imitación del modelo estadounidense de Clubes 4-H. Por su parte, el Programa de Mejoramiento del Hogar fue la adaptación local de los programas de Home Economics del Cooperative Extension Service. PICADO, 2012a. En la década de 1960, como mencionamos arriba, el SEA desarrolló en España los "Planteles de Jóvenes Rurales" e incorporó Agentes de Economía Doméstica a sus delegaciones (agencias) comarcales. 
quismo pretendía convertir a la agricultura en un sector productivo adaptado a los requerimientos del sistema de mercado de la posguerra mundial.

A pesar de nuestra vocación comparativa, esta investigación no quiere perder de vista la dimensión local (estatal o a escalas territoriales menores) de estos procesos históricos. Organismos económicos internacionales (el Banco Mundial, la FAO, la OECE o la OEA) y fundaciones privadas norteamericanas (Rockefeller o Ford) jugaron un papel rector en el impulso político del modelo de modernización agrícola y desarrollo económico que se deseaba consolidar en el bloque capitalista y exportar a sus áreas de influencia. Cabría preguntarse hasta qué punto fue viable su implantación en contextos sociales, culturales y productivos tan diversos y en qué medida se encontraron con resistencias por parte de las comunidades locales que se opusieron u obligaron a la negociación y reorientación de estos proyectos de transformación estructural.

Poner el acento en el estudio de las políticas agrarias del franquismo del desarrollismo y, particularmente, de la creación y actuación del Servicio de Extensión Agraria en el contexto de la difusión de la Teoría de la Modernización, puede colaborar en el análisis de una serie de procesos históricos interrelacionados (de cambio social, cultural, económico, político) que se revelan complejos y conflictivos. La propia construcción de dicha teoría de desarrollo, como adelantábamos al comienzo, se inscribe en una dinámica de conflicto bipolar entre dos sistemas ideológicos enfrentados. A su vez, las políticas concretas implementadas bajo la cobertura discursiva de esta teoría habrán de confrontar en cada contexto particular con otras maneras de entender la agricultura al margen del modelo hegemónico que se pretendía implementar.

\section{REFERENCIAS BIBLIOGRÁFICAS}

Álvaro Moya, Adoración

2011 "Guerra fría y formación de capital humano durante el franquismo. Un balance sobre el programa estadounidense de ayuda técnica, 1953-1963”. Historia del Presente. Madrid, $\mathrm{n}^{\circ}$ 17, pp. 13-25.

APTER, David

1972a "El sistema de movilización como prototipo de modernización". En APTER, Política de la modernización. Buenos Aires. Paidós, pp. 288-312.

1972b "Hacia una teoría de la modernización". En APTER, Política de la modernización. Buenos Aires. Paidós, pp. 21-52.

Banco Internacional de ReConstrucción y Fomento (BIRD)

1962 Informe del Banco Mundial. Oficina de Coordinación y Programación Económica.

Banco Internacional de Reconstrucción y Fomento (BIRD) - Food and Agriculture Organization of the United Nations (FAO)

1966 El desarrollo de la agricultura en España. Informe del Banco Internacional de Reconstrucción y Fomento y de la Organización de las Naciones Unidas para la Agricultura y la Alimentación. Madrid. Ministerio de Hacienda. 
Barciela, Carlos

2011 "Historia del Ministerio de Agricultura (1936-1965)". En Robledo (coord.), Historia del Ministerio de Agricultura 1900-2008. Política agraria y pesquera de España. Madrid. Ministerio de Medio Ambiente, Rural y Marino (MARM), pp. 145-221.

Barciela, Carlos - López Ortiz, María Inmaculada

2003 "El fracaso de la política agraria del primer franquismo, 1939-1959. Veinte años perdidos para la agricultura española". En BARCIELA (ed.), Autarquía y mercado negro: el fracaso económico del primer franquismo, 1939-1959. Barcelona. Crítica, pp. 55-94.

BENDIX, Richard

1966-67 "Tradition and Modernity reconsidered". Comparative Studies in Society and History. Cambridge, ${ }^{\circ}$ 9, pp. 292-346.

BRUNIER, Sylvain

2012 Conseillers et conseillères agricoles en France (1945-1983). L'amour du progrès aux temps de «la révolution silencieuse». Tesis de Doctorado inédita. Grenoble. Université de Grenoble.

Cabana Iglesia, Ana - Díaz-Geada, Alba

2010 "Agrarian technicians and the modernization of agriculture in Francoist Spain (1936-1975)”. En International Conference Rural History 2010. University of Sussex. 13-16 de septiembre de 2010.

2013 “Agrarian Fascism in Rural Spain (1936-1951)”. En FernándEZ-Prieto - PANMontojo - CABo (eds.), Agriculture in the age of Fascism. Authoritarian technocracy and rural modernization, 1922-1945. Turnhout. Brepols.

Calvo González, Óscar

2001 “Bienvenido, Míster Marshall! La ayuda económica americana y la economía española en la década de 1950". Revista de Historia Económica. Cambridge, $\mathrm{n}^{\mathrm{o}}$ extraordinario, pp. 253-275.

CARRERAS, Albert - TAFUnell, Xavier

2004 Historia Económica de la España Contemporánea. Barcelona. Crítica.

Cazorla, Antonio

2010 Fear and Progress. Ordinary Lives in Franco's Spain, 1939-1975. Chichester. Wiley-Blackwell.

Comín, Francisco - Hernández, Mauro - Llopis, Enrique

2002 Historia Económica de España. Siglos X-XX. Barcelona. Crítica.

DA SILVA, Claiton M.

2014 "El avance de la ciencia y la tecnología sobre las barreras de la lengua, la raza y las tradiciones: la Associação Americana Internacional (AIA) y los programas de modernización de la agricultura en Brasil”. En VIII Edición Encuentros por la Historia: Políticos, agrónomos y agricultores. Poder y espacios rurales iberoamericanos en el siglo XX. Costa Rica, Universidad Nacional. 
De Santiesteban, Fabiola

2009 "El desembarco de la Fundación Ford en España". Ayer. Madrid, n ${ }^{0}$ 75, pp. 159-191.

Delgado, Lorenzo

2003 "Las relaciones culturales entre España y Estados Unidos, de la Guerra Mundial a los Pactos de 1953". Cuadernos de Historia Contemporánea. Madrid, $\mathrm{n}^{\mathrm{o}} 25$, pp. 35- 59.

2012 "Objetivo: atraer a las élites. Los líderes de la vida pública y la política exterior norteamericana en España (1948-1960)”. En NiÑo - MonTERo (eds.), Guerra Fría y propaganda. Estados Unidos y su cruzada cultural en Europa y América Latina. Madrid. Biblioteca Nueva, pp. 235-276.

Delgado, Lorenzo - Elizalde, María Dolores (eds.)

2005 España y Estados Unidos en el Siglo XX. Madrid. CSIC.

Delgado, Lorenzo - León, Pablo (eds.)

2001 "El sueño americano. Americanización y franquismo". Historia del Presente. Madrid, $n^{\circ} 17$, pp. 5-70.

Díaz-GEADA, Alba

2013 Mudar en común. Cambios económicos, sociais e culturais no rural galego do franquismo e da transición, 1959-1982. Tesis de doctorado inédita. Santiago de Compostela. Universidade de Santiago de Compostela.

Díaz Geada, Alba - Lanero TÁboas, Daniel

2012 "Modernizing the Countryside: The Agrarian Extension Service in Francoist Spain (1955-1975)". En XIII World Congress of Rural Sociology. Lisboa. 29 de julio-4 de agosto de 2012.

2014a "A la sombra del amigo americano: sobre las raíces intelectuales y los agentes de la modernización de la agricultura en España (1955-1975)". En VIII Edición Encuentros por la Historia 2014, "Políticos, agrónomos y agricultores. Poder y espacios rurales latinoamericanos en el siglo XX”. Heredia. Universidad Nacional de Costa Rica. 19-22 mayo 2014.

2014b «Un modelo de modernización agraria: sobre la influencia intelectual del extensionismo americano en las políticas agrarias del tardofranquismo (19551975)». En XII Congreso Asociación Historia Contemporánea. Madrid. $17-$ 19 septiembre 2014.

Díaz-Geada, Alba - Lanero TÁboas, Daniel - Fernández-Prieto, Lourenzo - Cabana IGLESIA, Ana

2012 "Agricultural Extension Programmes in Postwar Europe: A Comparative Study of Two Extreme Cases - Spain and the Netherlands (1946-1973)". En IX European Social Science History Conference. Glasgow. 11-14 de abril de 2012.

Fernández, José A. - Picado, Wilson

2013 "Batallas sin guerra. Asistencia Técnica estadounidense y la modernización agrícola en América Latina de posguerra". En XIV Congreso Internacional de Historia Agraria. Badajoz. 7-9 de noviembre de 2013. 
FeRnÁNDEZ PRIETO, Lourenzo

2007 El apagón tecnológico del franquismo. Estado e innovación en la agricultura española del siglo XX. Valencia. Tirant-Lo Blanch - Ministerio de Cultura.

Fernández Prieto, Lourenzo - Pan-Montojo, Juan - Cabo, Miguel

2014 "Fascism and modernity in the European countryside: a global view". En Fernández Prieto - Pan-Montojo - Cabo (eds.), Agriculture in the Age of Fascism. Authoritarian Technocracy and Rural Modernization, 1922-1945. Turnhout. Bélgica, pp. 19-36.

García Apodaca, Anacleto

1953 Enseñando al agricultor. Enseñanza agrícola en un país latinoamericano. Tegucigalpa. American Embassy.

1958 "Las fundaciones filantrópicas y la ayuda técnica". Boletín Informativo. Servicio de Extensión Agrícola. Madrid, nº 2, pp. 7-8.

1959a "La participación del Servicio de Extensión Agrícola en el programa de intercambio técnico de la I.C.A.". Boletín Informativo. Servicio de Extensión Agraria. Madrid, no 14, p.19.

1959b La Extensión Agrícola en España (Documental). En: http://ruralmedia. eu/2009/05/la-extension-agricola-en-espana/

García Gutiérrez, José

1994 "Extensión Agraria: una labor de educación permanente en el mundo rural". Papeles de Economía Española, Madrid, nº 60/61, pp. 167-173.

Gómez Ayau, Emilio

1960 "Divulgación y extensión agrícola". Revista de Estudios Agrosociales. Madrid, no 30, pp. 33-64.

Gómez Benito, Cristóbal

1996 Políticos, burócratas y expertos: un estudio de la política agraria y la sociología rural en España (1936 -1959). Madrid. Siglo XXI.

Gómez Benito, Cristóbal - Luque Pulgar, Emilio

2007a "Modernización agraria, modernización administrativa y franquismo. El modelo educativo y administrativo del Servicio de Extensión Agraria (19551986)". Areas. Revista Internacional de Ciencias Sociales. Murcia, nº 26, pp. 13-29.

2007b Semillas de modernidad. El Servicio de Extensión Agraria y la transformación del campo español (1955-1980). Madrid. Ministerio de Agricultura, Pesca y Alimentación.

Guirao PiñeYro, Fernando

1998 Spain and the Reconstruction of Western Europe, 1945-57. Challenge \& Response. London \& New York. Macmillan Press \& St. Martin's Press.

HARWOOD, Jonathan

2009 "Peasant friendly plant breeding and the early years of Green Revolution in Mexico". Agricultural History. Cambridge, vol. 83, nº 3, pp. 384-410. 
HERnÁNDEZ Burgos, Claudio

2013 Franquismo a ras de suelo. Zonas grises, apoyos sociales y actitudes durante la dictadura (1936-1976). Granada. Editorial Universidad de Granada.

HERNANDO VARELA, J.

2001 "El proyecto de investigación agrícola del Banco Mundial y la modernización de la investigación agraria en España en la década de los setenta". Estudios Agrosociales y Pesqueros. Madrid, nº 190, pp. 43-63.

HewitT de Alcántara, Cynthia

1978 La modernización de la agricultura mexicana (1940-1970). México. Siglo XXI.

Hodge, Joseph M.

2007 Triumph of the Expert: Agrarian Doctrines of Development and the Legacies of British Colonialism. Athens. Ohio University Press.

2010 “The British 'School' of Tropical Agriculture: approaches, debates and legacies". International Conference Rural History 2010. University of Sussex. 13-16 de septiembre.

Huntington, Samuel

1968 Political Order in Changing Societies. New Haven. Yale University Press.

1976 The Change to Change: Modernization, development and politics. New York. Free Press.

KAREL, Erwin

2006 "Modelling the farm-family, 1953-1970". En VI European Social Science History Conference. Amsterdam. 22-25 de marzo de 2006.

2010 "Modernization of the Dutch agriculture system, 1950-2000". En Rural History Conference. University of Sussex. Brighton. 13-16 de septiembre de 2010.

Lamo de Espinosa Y EnRíquez de NaVARRa, Emilio

1962 "El Informe del Banco Mundial y la Agricultura Española". Revista de Estudios Agrosociales. Madrid, no 41, pp. 11-54.

LEÓN, Pablo

2009 "Los canales de la propaganda norteamericana en España, 1945-1960". Ayer. Madrid, $\mathrm{n}^{\circ} 75$, pp. 133-158.

2012 "Faith in the USA. El mensaje de la diplomacia pública americana en España (1948-1960)". En NiÑo - MonTERo (eds.), Guerra Fría y propaganda. Estados Unidos y su cruzada cultural en Europa y América Latina. Madrid. Biblioteca Nueva, pp. 197-234.

Martín GarCía, Julio

2012 "Semillas de modernidad. El Servicio de Extensión Agraria y la transformación del campo español”. Padrón, pp. 127-131.

MAUNDER, Addison $\mathrm{H}$.

1966 "Why an Extension Service Today in Other Countries?". En SANDERS - ARbour - Clark - Jones, The Cooperative Extension Service. Nueva Jersey. Prentice-Hall. 
Mínguez Goyanes, Luis

1997 "La Iglesia española y la ayuda social americana (1954-1968)". Hispania Sacra. Madrid, no 49, pp. 421-462.

Molinero, Carme - Ysás, Pere

2008 La anatomía del franquismo. De la supervivencia a la agonía, 1945-1977. Barcelona. Crítica.

MOSHER, Arthur T.

1957 Technical co-operation in Latin-American Agriculture. Chicago. The University of Chicago Press.

Niño, Antonio (ed.)

2009 "La ofensiva cultural norteamericana durante la Guerra Fría". Ayer. Madrid, $\mathrm{n}^{\mathrm{o}}$ 75, pp. 13-221.

Niño, Antonio - Montero, José Antonio (eds.)

2012 Guerra Fría y propaganda. Estados Unidos y su cruzada cultural en Europa y América Latina. Madrid. Biblioteca Nueva.

PicADo, Wilson

2011 "Breve historia semántica de la Revolución Verde". En LANERo TÁBoAs FreIRE (coords.), Agriculturas e innovación tecnológica en la península ibérica (1946-1975). Madrid. Ministerio de Medio Ambiente - Medio Rural y Marino, pp. 25-50.

2012a Conexiones de la Revolución Verde. Estado y cambio tecnológico en la agricultura de Costa Rica durante el periodo 1940-1980. Tesis de doctorado inédita. Santiago de Compostela. Universidade de Santiago de Compostela.

2012b "En busca de la genética guerrera. Segunda Guerra Mundial, cooperación agrícola y Revolución Verde en la agricultura de Costa Rica”. Historia Agraria. Murcia, $\mathrm{n}^{\circ}$ 56, pp. 107-134.

Puig, Nuria

2003 "La ayuda económica americana y los empresarios españoles". Cuadernos de Historia Contemporánea. Madrid, nº 25, pp. 109-129.

Ramos Gorostiza, José Luis - Pires Jiménez, Luis

2009 "Los economistas españoles ante la planificación indicativa del desarrollismo". En De La Torre - García-ZúÑIGA (eds.), Entre el Mercado y el Estado. Los planes de desarrollo durante el franquismo. Pamplona. Universidad Pública de Navarra.

Rodríguez, Miguel

2012 "La perspectiva latinoamericana de la potencia cultural estadounidense". En Niño - Montero (eds.), Guerra Fría y propaganda. Estados Unidos y su cruzada cultural en Europa y América Latina. Madrid. Biblioteca Nueva, pp. 277-309.

RodRíGUEZ JiMÉnEZ, Francisco Javier

2010 ¿Antídoto contra el antiamericanismo? American Studies en España, 19451969, Valencia, PUV. 
RodríGuez Troncoso, Amador

2009 "Vivencias de un agente de Extensión". En Rodríguez Troncoso - García Gutiérrez - García Fernández, El Servicio de Extensión Agraria. Vivencias, recuerdos y vigencia. Madrid. Centro de Publicaciones del Ministerio de Medio Ambiente, Rural y Marino (MARM), pp. 15-146.

Rogers, Everett

1969 Modernization among peasants. New York. Holt. Rinehart and Winston.

Rollo, María Fernanda

2011 "Ambiciones frustradas: las vías de modernización y el programa de asistencia técnica americana a la agricultura portuguesa en la posguerra (1948-1956)". En Lanero TÁboas - Freire (coords.), Agriculturas e innovación tecnológica en la Península Ibérica (1946-1975). Madrid. Centro de Publicaciones del Ministerio de Medio Ambiente, Medio Rural y Marino (MARM), pp. 107-133.

Rostow, Walter

1961 Las etapas del crecimiento económico: un manifiesto no comunista. México. Fondo de Cultura Económica.

Sánchez De Puerta Trujillo, Fernando

1996 Extensión agraria y desarrollo rural. Sobre la evolución de las teorías y praxis extensionistas. Madrid. MAPA, pp. 913-948.

1997 "Estado y agricultura: La extensión agraria”. En Gómez De BenITo - GonzÁLEZ RodríGuez (eds.), Agricultura y sociedad en la España contemporánea. Madrid. MAPA.

SANDERs, H. C. (ed.)

1966 The Cooperative Extension Service. New Jersey. Prentice Hall, Inc.

ScotT-Smith, Giles

2012 "Las élites de Europa Occidental y el Foreign Leader Program (1949-1969)". En NiÑo - Montero (eds.), Guerra Fría y Propaganda. Estados Unidos y su cruzada cultural en Europa y América Latina. Madrid. Biblioteca Nueva, pp. 123-154.

SHIVA, Vandana

1991 The violence of the Green Revolution. Third World Agriculture, Ecology and Politics. Penang. Third World Network.

Termis Soto, Fernando

2005 Renunciando a todo. El régimen franquista y los Estados Unidos desde 1945 hasta 1963. Madrid. Biblioteca Nueva - UNED.

VIÑAS, Ángel

2003 En las garras del águila. Los pactos con Estados Unidos, de Francisco Franco a Felipe González (1945-1995). Barcelona. Crítica.

Zorrilla Dorronsoro, Ángel

1962 "Inversiones en colonización". Revista de Estudios Agrosociales. Madrid, n 41, pp. 61-78. 